\title{
Diagnosis of acute mononucleosis in emergency: comparison of rapid tests
}

\author{
Federica Scaggiante', Sonia Moratelli', Patrizia Rossi², Clara Larcher², Martin Ogriseg' \\ I Laboratorio di Patologia Clinica, Comprensorio Sanitario di Bressanone, Azienda Sanitaria dell'Alto Adige \\ 2 Laboratorio Aziendale di Microbiologia e Virologia, Comprensorio Sanitario di Bolzano, Azienda Sanitaria dell'Alto Adige
}

Key words: EBV, monotest, acute mononucleosis, EBV-specific rapid test

Diagnosi di mononucleosi infettiva acuta in regime di urgenza: test rapidi a confronto

\section{SUMMARY}

Epstein-Barr virus (EBV) is a gammaherpesvirus that causes a number of clinical syndromes, including acute mononucleosis. Acute infection with EBV can vary widely with regard to the severity and presentation of illness, ranging from an asymptomatic infection to a serious, life-threatening version of mononucleosis with associated liver damage and splenomegaly. Additionally, other acute viral syndromes, including those caused by hepatitis viruses and cytomegalovirus (CMV), can lead to similar clinical syndromes. The variety of symptoms and the overlap with other viral infections underscore the importance of laboratory testing in the diagnosis of acute EBV-related disease.The purpose of this study was to evaluate the utility of an agglutination test for the detection of heterophile antibodies (Monotest) and two EBV-specific rapid immunochromatographic tests (VCA-lgM and VCA-lgG/EBNA-lgG). Heterophile antibody determination is resulted to have not a real diagnostic utility for the low sensibility and specificity of the test. In our experience the only use of VCA-IgG/EBNA-lgG test is sufficient to discriminate between an acute mononucleosis and a past infection.

\section{INTRODUZIONE}

La mononucleosi infettiva acuta è una sindrome virale che ha come agente eziologico il virus di Epstein-Barr (EBV), appartenente alla famiglia degli herpesviridae, sottofamiglia gammaherpesvirinae.

L'infezione primaria da EBV, che generalmente decorre in modo asintomatico in soggetti immunocompetenti, causa la malattia per lo più in giovani adulti, con sintomi quali tonsilliti, faringiti, ingrossamento dei linfonodi cervicali, febbre moderata, a volte dolori addominali, epatopatie. Nella maggioranza dei casi l'infezione primaria da EBV è accompagnata da aumento degli enzimi epatici e dei linfociti circolanti, in gran parte atipici (mononuclear cells).

I segni clinici della mononucleosi infettiva sono provocati dalla massiccia risposta immunitaria che coinvolge i linfociti $\mathrm{T}$ citotossici diretti contro le cellule infettate da EBV. La diagnosi di mononucleosi infettiva acuta in regime di urgenza viene solitamente effettuata sulla base della sintomatologia del paziente e della presenza di anticorpi eterofili circolanti e/o di forme linfocitarie atipiche. In ragione del fatto che la sintomatologia risulta simile in altre sindromi di origine virale con agenti eziologici quali Citomegalovirus (CMV), Herpesvirus 6 (HHV6), Adenovirus,
Paramyxovirus, Orthomyxovirus, che in molti pazienti non sono riscontrabili forme linfocitarie atipiche e che il test per la ricerca di anticorpi eterofili ha una bassa sensibilità e specificità, tale diagnosi può rivelarsi poco accurata.

\section{Scopo}

Scopo di questo lavoro è stato valutare l'utilità di test rapidi per la ricerca di anticorpi diretti verso antigeni virali specifici per EBV, in particolare un test rapido per la ricerca dei soli anticorpi di fase acuta diretti verso l'antigene capsidico (VCA$\operatorname{IgM})$ e un test rapido per la simultanea ricerca di anticorpi della classe IgG precoci (VCA-IgG) e tardivi (EBNA-IgG).

Si è inoltre valutato se fosse possibile sostituire il test di agglutinazione per la ricerca di anticorpi eterofili (Monotest), attualmente utilizzato nella diagnosi di mononucleosi infettiva acuta in regime di urgenza, con solo uno dei due test rapidi valutati o con entrambi come da indicazione della ditta produttrice.

\section{MATERIALI E METODI}

Sono stati raccolti in modo prospettico e randomizzato 30 sieri di pazienti con richiesta urgente di test diagnostici per sospetta mononucleosi infettiva acuta. Su siero fresco è stato eseguito il

\section{Corresponding author: Federica Scaggiante}

Azienda Sanitaria dell'Alto Adige, Comprensorio Sanitario di Bressanone, Laboratorio di Patologia Clinica Via Dante 5I, 39042 Bressanone (BZ) - Tel. 0472-8I3200, Fax 0472-8I3209

E-mail: fscaggiante@as-bressanone.it 
test di agglutinazione per la ricerca di anticorpi eterofili (Monotest: Cellognost-Mononucleosis, Siemens), come da normale procedura.

I sieri sono stati quindi conservati a $-20^{\circ} \mathrm{C}$ e analizzati a distanza di un massimo di 30 giorni.

Sono stati eseguiti, come da metodica, un test rapido immunocromatografico per la ricerca di VCA-IgM (TruEBV-M, Meridian) e un test rapido immunocromatografico per la simultanea ricerca di VCA-IgG/EBNA-IgG (TruEBV-G, Meridian).

È stato possibile confermare un'infezione acuta da EBV con presenza di una banda di positività per VCA-IgM nel test TruEBV-M (Figura I) e con presenza della sola banda di positività per le VCA-IgG nel test TruEBV-G; per quest'ultimo la presenza di positività per entrambi gli anticorpi di classe IgG (VCA ed EBNA) o delle sole EBNA-IgG indicava invece un'infezione pre-

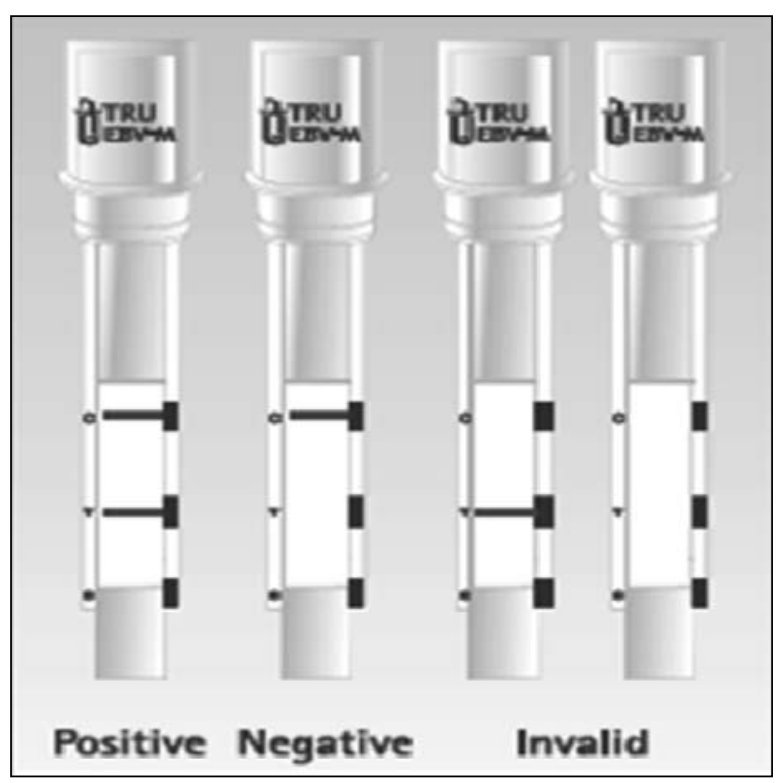

Figura I. Interpretazione risultati per TruEBV-M.

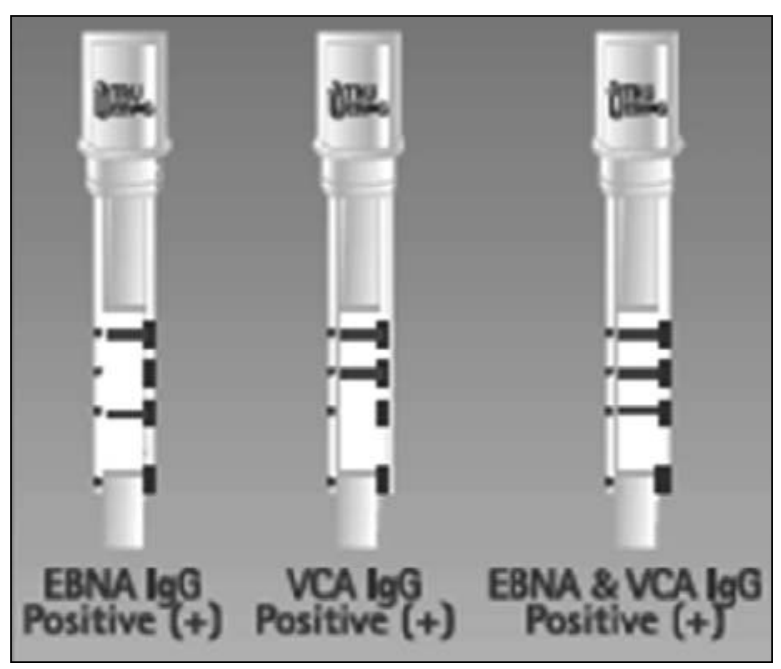

Figura II. Interpretazione risultati per TruEBV-G. gressa (Figura II).

I sieri sono stati successivamente inviati al Laboratorio Aziendale di Microbiologia e Virologia di Bolzano e testati con metodo di riferimento ELISA in micropiastra (kit Novagnost su strumento BEP III, Siemens) per l'intero profilo sierologico EBV (VCA-IgM, VCA-IgG, EA-IgG ed EBNA-IgG). Si rilevava un'infezione acuta in caso di assenza di anticorpi IgG-EBNA e presenza di almeno un'anticorpo diretto verso antigeni virali precoci (EA o VCA).

\section{RISULTATI}

In 22 pazienti c'è stata concordanza di risultato con tutte le metodiche testate: 6 pazienti con risposta anticorpale negativa e quindi suscettibili, 15 pazienti con infezione da EBV pregressa e un paziente con infezione acuta.

In 3 pazienti si è evidenziata un'infezione acuta da EBV con i test TruEBV-M e TruEBV-G, ma non con Monotest; il metodo di riferimento ha confermato la presenza di un'infezione acuta.

In 2 pazienti è stata riscontrata un'infezione da EBV pregressa con i test TruEBV-M e TruEBV-G, mentre il Monotest indicava un'infezione acuta; il metodo di riferimento ha confermato la presenza di un'infezione pregressa.

In 2 pazienti è stata rilevata un'infezione acuta da EBV con Monotest e TruEBV-G, ma non con TruEBV-M; la metodica di riferimento ha confermato un'infezione acuta.

In un paziente si è evidenziata una negatività per Monotest e un'infezione pregressa con TruEBV$\mathrm{G}$, mentre il test TruEBV-M indicava un'infezione acuta; il metodo di riferimento ha confermato un'infezione pregressa.

\section{DISCUSSIONE E CONCLUSIONI}

Il Monotest ha evidenziato un'infezione acuta in soli 3 casi su 6 riscontrati; in 2 pazienti ha dato un risultato falso positivo. Il test TruEBV-M ha rilevato un'infezione acuta in 4 casi su 6 ; in un paziente ha dato un risultato falso positivo.

Il test TruEBV-G ha confermato un'infezione acuta da EBV in tutti i 6 casi riscontrati e non ha dato risultati falsi positivi.

Si deduce pertanto che il Monotest è una metodica troppo poco specifica e sensibile per poter avere una reale utilità diagnostica.

Tra i test rapidi per la ricerca di anticorpi specifici per EBV, inoltre, quello che ha migliore sensibilità e specificità risulta essere il TruEBV-G, che dà in aggiunta la possibilità di discriminare tra un'infezione pregressa e una condizione di suscettibilità.

Va considerato inoltre che il paziente che ricorre alle cure mediche per sospetta mononucleosi 


\begin{tabular}{|c|c|c|c|c|c|c|c|c|c|}
\hline & \multicolumn{4}{|c|}{ test rapidi } & \multicolumn{4}{|c|}{ micropiastra } & \multirow{3}{*}{$\begin{array}{c}\text { stato } \\
\text { sierologico }\end{array}$} \\
\hline & \multirow{2}{*}{ mono-test } & \multirow{2}{*}{ EBV-M } & \multicolumn{2}{|c|}{ EBV-G } & \multirow{2}{*}{ VCA IgG } & \multirow{2}{*}{ VCA IgM } & \multirow{2}{*}{ EBNA } & \multirow{2}{*}{ EA-IgG } & \\
\hline & & & VCA & EBNA & & & & & \\
\hline 1 & NEG & NEG & POS & POS & POS & NEG & POS & NEG & $\mathrm{P}$ \\
\hline 2 & NEG & NEG & POS & POS & POS & NEG & POS & POS & $\mathrm{P}$ \\
\hline 3 & POS & NEG & POS & POS & POS & NEG & POS & NEG & $\mathrm{P}$ \\
\hline 4 & NEG & POS & POS & POS & POS & NEG & dubbio & NEG & $P$ \\
\hline 5 & POS & NEG & POS & POS & POS & NEG & POS & NEG & $\mathrm{P}$ \\
\hline 6 & NEG & POS & POS & NEG & POS & POS & NEG & POS & A \\
\hline 7 & NEG & NEG & NEG & NEG & NEG & NEG & NEG & NEG & $N$ \\
\hline 8 & NEG & NEG & POS & POS & POS & NEG & POS & NEG & $P$ \\
\hline 9 & NEG & NEG & POS & POS & POS & NEG & POS & NEG & $\mathrm{P}$ \\
\hline 10 & NEG & NEG & NEG & NEG & NEG & NEG & NEG & NEG & $\mathrm{N}$ \\
\hline 11 & NEG & NEG & POS & POS & POS & NEG & POS & NEG & $P$ \\
\hline 12 & NEG & NEG & NEG & NEG & NEG & NEG & NEG & NEG & $\mathrm{N}$ \\
\hline 13 & NEG & NEG & POS & POS & POS & NEG & POS & NEG & $P$ \\
\hline 14 & NEG & NEG & POS & POS & POS & NEG & POS & NEG & $\mathrm{P}$ \\
\hline 15 & NEG & POS & POS & NEG & POS & POS & NEG & NEG & A \\
\hline 16 & NEG & NEG & POS & POS & POS & NEG & POS & NEG & $\mathrm{P}$ \\
\hline 17 & NEG & POS & POS & NEG & POS & POS & NEG & POS & A \\
\hline 18 & NEG & NEG & POS & POS & POS & NEG & POS & NEG & $P$ \\
\hline 19 & NEG & NEG & NEG & NEG & NEG & NEG & NEG & NEG & $\mathrm{N}$ \\
\hline 20 & NEG & NEG & NEG & POS & POS & NEG & POS & POS & $\mathrm{P}$ \\
\hline 21 & NEG & NEG & NEG & NEG & NEG & NEG & NEG & NEG & $\mathrm{N}$ \\
\hline 22 & NEG & NEG & POS & POS & POS & NEG & POS & dubbio & $\mathrm{P}$ \\
\hline 23 & NEG & NEG & NEG & NEG & NEG & NEG & NEG & NEG & $\mathrm{N}$ \\
\hline 24 & NEG & NEG & POS & POS & POS & NEG & NEG & NEG & $P$ \\
\hline 25 & NEG & NEG & POS & POS & POS & NEG & POS & POS & P \\
\hline 26 & NEG & NEG & POS & POS & POS & NEG & POS & NEG & $P$ \\
\hline 27 & NEG & NEG & NEG & POS & POS & NEG & POS & NEG & $P$ \\
\hline 28 & POS & NEG & POS & NEG & POS & POS & NEG & POS & A \\
\hline 29 & POS & NEG & POS & NEG & dubbio & POS & NEG & POS & A \\
\hline 30 & POS & POS & POS & NEG & POS & POS & NEG & POS & A \\
\hline
\end{tabular}

Tabella riassuntiva dei risultati ottenuti: sono evidanziati in grigio i pazienti per quali è stata riscontrata discordanza di risultato di almeno uno dei test rapidi rispetto al metodo di riferimento (ELISA in micropiastra). Interpretazione dello stato sierologico: $A$ = infezione acuta; $P=$ infezione pregressa; $N=$ condizione di suscettibilità.

infettiva acuta è di norma già sintomatico e ha quindi superato la situazione sierologica di sola presenza di anticorpi di fase acuta (IgM).

Nel nostro studio, infatti, non è stato riscontrato alcun caso di presenza di soli anticorpi VCA-IgM. Possiamo pertanto affermare che per evidenziare un'infezione acuta da EBV, il test immunocromatografico TruEBV-G fornisce ottimi risultati, anche senza l'esecuzione aggiuntiva del test TruEBV-M.

Tuttavia si sottolinea l'importanza di ampliare la casistica dei pazienti al fine di confermare $\mathrm{i}$ promettenti risultati del test immunocromatografico.

\section{BIBLIOGRAFIA}

1. Godshall SE, Kirchner JT. Infectious mononucleosis. Complexities of a common syndrome. Postgraduate medicine, Jun. 2000; 107: 175-9; 183-4, 186.

2. Hess RD. Routine Epsein-Barr Virus Diagnostics from the Laboratory Perspective: Still Challenging after 35 Years. Journal of Clinical Microbiology, Aug. 2004; 3381-7.

3. Klutts JS, et al. Evidence-Based Approach for Interpretation of Epstein-Barr Virus Serological Patterns. Journal of Clinical Microbiology, Oct. 2009; 3204-10.

4. Svahn A, et al. Evaluation of three commercial enzyme-linked immunosorbent assays and two latex agglutination assays for diagnosis of primary EpsteinBarr virus infection. Journal of Clinical Microbiology. Nov. 1997; 35: 2728-32. 